\title{
Conhecimento dos profissionais de enfermagem sobre cuidados paliativos
}

\author{
Knowledge of nursing professionals about palliative care \\ Conocimiento de profesionales de enfermeíra sobre cuidados paliativos
}

Recebido: 22/06/2021 | Revisado: 30/06/2021 | Aceito: 03/07/2021 | Publicado: 15/07/2021

\author{
Isabela Pechinim \\ ORCID: https://orcid.org/0000-0002-3927-9323 \\ Faculdade de Medicina de São José do Rio Preto, Brasil \\ E-mail: isabelapechinim@gmail.com \\ Lara Fernanda da Silva Moraes \\ ORCID: https://orcid.org/0000-0001-9614-3142 \\ Faculdade de Medicina de São José do Rio Preto, Brasil \\ E-mail: laramoraes0612@gmail.com \\ Maria Helena Pinto \\ ORCID: https://orcid.org/0000-0003-0601-8511 \\ Faculdade de Medicina de São José do Rio Preto, Brasil \\ E-mail: helmarpi@gmail.com \\ Giovana Spina \\ ORCID: https://orcid.org/0000-0001-9275-4317 \\ Centro Universitário Padre Albino, Brasil \\ E-mail: giovanaspina@gmail.com
}

\begin{abstract}
Resumo
O objetivo deste estudo foi avaliar o conhecimento da equipe de enfermagem sobre Cuidados Paliativos (CP) em Unidades Básicas de Saúde e Estratégia da Família (UBSF) e unidades de internação hospitalar. Trata-se de um estudo transversal, desenvolvido nas unidades de internação de um hospital público e duas UBSF, do interior do estado de São Paulo. Participaram do estudo, doze enfermeiros e cinco auxiliares/técnicos de enfermagem da UBSF e dez enfermeiros e 43 auxiliares/técnicos do hospital, totalizando 70 profissionais que atuavam nas unidades há 12 meses ou mais. Os dados foram coletados por meio de dois questionários, sendo um com dados sociodemográficos e outro com 30 afirmações sobre $\mathrm{CP}$, dados que foram analisados de forma descritiva e inferencial. Constatou-se que $76,66 \%$ dos profissionais acertaram as questões de conhecimentos gerais sobre CP. Apenas $18,75 \%$ dos auxiliares/técnicos de enfermagem e 36,36\% dos enfermeiros acertaram a definição de distanásia e de ortotanásia $70,83 \%$ dos auxiliares/técnicos e 54,55\% dos enfermeiros. Há tendência estatística entre o número de acertos com o tempo de experiência em CP e aqueles que fizeram curso específico de CP. Concluiu-se que aspectos básicos do cuidado paliativo devem fazer parte da educação em serviço; é importante que os profissionais entendam que o plano de cuidados não deve abreviar a vida, mas oferecer qualidade de vida aos pacientes até o último momento da sua vida. Acredita-se que este estudo possa contribuir para uma reflexão sobre o ensino-aprendizagem e a formação do profissional da enfermagem.
\end{abstract}

Palavras-chave: Conhecimento; Profissionais de enfermagem; Cuidados paliativos.

\begin{abstract}
The aim of this study was to assess the knowledge of the nursing staff about palliative care (PC) in Basic Health and Family Strategy Units and hospital inpatient units. This is a cross-sectional study, developed in the inpatient units of a public hospital and two Basic Health Family Strategy Units, in a country town of São Paulo. Twelve nurses and five nursing assistants/technicians from Basic Health Family Strategy Units and ten nurses and 43 hospital assistants/technicians participated in the study, totaling 70 professionals who had worked in the units for 12 months or more. Data were collected through two questionnaires, one with sociodemographic data and the other with 30 statements about PC, data that were analyzed in a descriptive and inferential way. It was found that $76.66 \%$ of the professionals got the questions of general knowledge about PC. Only $18.75 \%$ of the nursing assistants/technicians and $36.36 \%$ of the nurses agreed on the definition of dysthanasia and orthothanasia, $70.83 \%$ of the assistants/technicians and $54.55 \%$ of the nurses. There is a statistical trend between the number of correct answers with the time of experience and those who took a specific PC course. It was concluded that basic aspects of palliative care should be part of in-service education; It is important for professionals to understand that the care plan should not shorten life, but offer quality of life to patients until the last moment of their life. It is believed that this study can contribute to a reflection on teaching-learning and professional training.
\end{abstract}

Keywords: Knowledge; Nursing professionals; Palliative care. 


\begin{abstract}
Resumen
El objetivo de este estudio fue evaluar el conocimiento del personal de enfermería sobre Cuidados Paliativos (CP) en Unidades Básicas de Salud y Estrategia Familiar (UBSF) y unidades de internación hospitalaria. Se trata de un estudio transversal, desarrollado en las unidades de internación de un hospital público y dos UBSF, en el interior del estado de São Paulo. En el estudio participaron 12 enfermeros y cinco auxiliares / técnicos de enfermería de la UBSF y diez enfermeros y 43 auxiliares / técnicos hospitalarios, totalizando 70 profesionales que habían trabajado en las unidades durante 12 meses o más. Los datos fueron recolectados a través de dos cuestionarios, uno con datos sociodemográficos y otro con 30 declaraciones sobre $\mathrm{CP}$, datos que fueron analizados de manera descriptiva e inferencial. Se encontró que el 76,66\% de los profesionales acertaron las preguntas de conocimientos generales sobre CP. Sólo el $18,75 \%$ de los auxiliares / técnicos de enfermería y el 36,36\% de las enfermeras coincidieron en la definición de distanasia y ortotanasia, el 70,83\% de los auxiliares / técnicos y el 54,55\% de las enfermeras. Existe una tendencia estadística entre el número de respuestas correctas con el tiempo de experiencia en CP y los que tomaron un curso específico de CP. Se concluyó que los aspectos básicos de los cuidados paliativos deberían formar parte de la educación en el servicio; Es importante que los profesionales comprendan que el plan de cuidados no debe acortar la vida, sino ofrecer calidad de vida a los pacientes hasta el último momento de su vida. Se cree que este estudio puede contribuir a una reflexión sobre la enseñanza-aprendizaje y la formación profesional.
\end{abstract}

Palabras clave: Conocimiento; Profesionales de enfermera; Cuidados paliativos.

\title{
1. Introdução
}

A Organização Mundial de Saúde (OMS) em 1990 definiu o conceito de Cuidados Paliativos (CP) e redefiniu em 2002, como "uma abordagem que promove qualidade de vida de pacientes e familiares que enfrentam doenças que ameaçam a vida, por meio da prevenção e alívio do sofrimento, avaliação e tratamento da dor e de outros problemas de natureza biopsicossocial e espiritual" (Matsumoto, 2012, p.26). Com uma visão ampliada, o CP se apresenta como uma abordagem multiprofissional especializada para dar suporte ao paciente e seus familiares, a fim de prevenir os sintomas e complicações da doença de base, controlar a dor e contribuir para a qualidade de vida, autonomia e sobrevida do paciente, e deve ser oferecido em todos os níveis de atenção (Silva et al., 2016).

Ressalta-se que o CP tem como objetivo melhorar a qualidade de vida dos usuários, de seus familiares e de seus cuidadores (D’Alessandro et al., 2020), onde o paciente que necessita desse cuidado estiver, seja no domicilio, na instituição hospitalar, no ambulatório, instituição de longa permanência, ou hospice (Worldwide Palliative Care Alliance, 2014). Além disto, possui alguns princípios norteadores importantes, como o início precoce do tratamento e acompanhamento, análise do cliente de forma integral com a inclusão de aspectos psicossociais e espirituais, a investigação adequada para a assistência e manejo dos sintomas, avaliação e reavaliação no alívio da dor e desconforto, oferta de suporte à família durante o processo de cuidado, reafirmação da importância da vida e a compreensão da morte como um processo intrínseco ao ser humano e natural, sem antecipar nem postergá-la (D’Alessandro et al., 2020). Neste contexto, cabe ao profissional de saúde ponderações éticas, incluindo a necessidade de ter conhecimento sobre conceitos de distanásia, eutanásia e ortotanásia, envolvendo princípios de bioética, como a de não maleficência ao paciente (Mota et al., 2021).

A distanásia é a intervenção de manter a vida, com ações médicas desproporcionais que tornam a morte algo doloroso, que causa sofrimento ao paciente e seus familiares, propiciando insucesso na recuperação da vida e condições de bem-estar (Silva, 2018). A eutanásia, por sua vez, é a interrupção intencional da vida, com a finalidade de evitar o sofrimento do indivíduo enfermo, o que no Brasil é ilegal, caracterizada como homicídio e a ortotanásia é a morte em seu processo natural, no momento certo, que respeita os direitos do indivíduo e seus desejos de modo que possa morrer com dignidade (Cano et al., 2020).

O enfermeiro oferece um cuidado com base no processo de enfermagem, considerando importantes ações, como educação em saúde, apoio emocional e social, avaliação, identificação de problemas e diagnóstico de enfermagem (Silva, 2021). A equipe de enfermagem possui papel fundamental em CP, visto que, os profissionais da categoria desenvolvem diversas ações beira leito e preconizam um vínculo maior com os pacientes promovendo a partir da assistência, o alívio da dor, 
dignidade e humanização (Steel et al., 2016). Além disso, a família é um importante aliado, pois pode favorecer a comunicação com o paciente, sendo que se adequada, harmoniosa e centrada nas necessidades do paciente, pode resultar em contribuições essenciais para o controle de desconfortos, minimizando o sofrimento de pacientes e demais familiares (Côbo et al., 2019).

No Brasil, a Resolução no. 41 (2018) normatizou os CP no Sistema Único de Saúde (SUS) permitindo que as redes de Atenção Primária à Saúde (APS) assegurem e promovam esses cuidados, uma vez que o CP teve nos últimos anos um enfoque maior em âmbito hospitalar, ressaltando que é na APS o primeiro contato da sociedade com os serviços de saúde. Para essa integração em rede cabe aos governos proporcionar que os CP estejam inseridos em todos os âmbitos de atenção à saúde. A frente disso destaca-se que é fundamental que a equipe tenha competências e habilidades para prestar cuidados básicos e que tenha a inclusão de profissionais especializados em CP, com a colaboração de instituições de ensino, como universidades para capacitação e pesquisa sobre o tema, além da educação em serviço e inclusão do tema no processo de formação destes profissionais (Silva et al., 2016).

Assim, é imprescindível verificar os conhecimentos dos profissionais, para o seu aperfeiçoamento e colaboração para uma assistência de qualidade, com foco na continuidade do cuidado oferecido, visando alcançar e melhorar os resultados da assistência, uma vez que os CP constituem uma área pouco explorada, principalmente na graduação e ensino técnico, apesar dos diversos avanços. Portanto, o objetivo deste estudo foi avaliar o conhecimento da equipe de enfermagem sobre cuidados paliativos em Unidades Básicas de Saúde e Estratégia da Família (UBSF) e Unidades de Internação Hospitalar.

\section{Metodologia}

Trata-se de um estudo descritivo, do tipo transversal, de natureza quantitativa. O estudo foi desenvolvido nas unidades de internação geriátrica, cirúrgica e clínica de um hospital de ensino do interior do estado de São Paulo, abrangendo todos os turnos de trabalho e duas Unidades Básicas de Saúde de Estratégia da Família (UBSF) da mesma cidade.

A população do estudo foi constituída de 120 profissionais de enfermagem, de ambos os sexos, que faziam parte do quadro de profissionais das instituições há 12 meses ou mais. A coleta de dados foi realizada entre os meses de outubro e dezembro de 2020 nos locais de trabalho por meio de dois instrumentos de coleta dos dados, que foram entregues aos profissionais dentro de um envelope, com o compromisso de entregarem preenchido após uma semana. Foram excluídos da pesquisa 48 profissionais que não entregaram os questionários preenchidos e dois que tinham tempo de trabalho menor que doze meses. Portanto, a amostra foi constituída de 70 profissionais da enfermagem, 22 enfermeiros e 48 auxiliares/técnicos sendo doze enfermeiros da UBSF e dez do hospital, cinco auxiliares/técnicos de enfermagem da UBSF e 43 do hospital.

Foi utilizado um questionário autoaplicável, elaborado pelos autores com base no Manual de Cuidados Paliativos, contendo 43 questões agrupadas em seis questões com dados sociodemográficas (sexo, faixa etária, categoria profissional, tempo de profissão, tempo de experiência em cuidados paliativos e local de trabalho); cinco questões com respostas "sim" ou "não" relacionadas a vivências dos profissionais com pacientes em CP: "Você tem experiência com pacientes em cuidados paliativos? Quantos anos de experiência?", "Você já vivenciou o processo de morrer/morte com alguém próximo?", "Você possui algum curso específico de Cuidados Paliativos?" e "Você se considera capaz para prestar cuidados a pacientes em cuidados paliativos?". Três questões de autoavaliação sobre cuidados paliativos também com respostas de "sim" e "não, e 30 afirmativas objetivas sobre cuidados paliativos, correspondendo a respostas "Verdadeiro", "Falso" ou "Não sei", entre elas quatro questões relacionadas a definições de cuidados paliativos (Q1), Eutanásia (Q5), Distanásia (Q6) e Ortotanásia (Q7).

O projeto foi aprovado pelo Comitê de Ética em Pesquisa da Faculdade de Medicina São José do Rio Preto (FAMERP), parecer $\mathrm{n}^{\circ}$ 4.096.251, de 10/05/2020. Todos os profissionais de enfermagem participantes assinaram o Termo de Consentimento Livre e Esclarecido após esclarecimentos sobre os procedimentos, objetivo e destino dos dados da pesquisa, 
com a garantia do sigilo dos dados, conforme a resolução 466/2012 do Conselho Nacional de Saúde, do Ministério da Saúde, que dispõe as diretrizes e normas regulamentares da pesquisa envolvendo seres humanos.

Os dados coletados foram tabulados e analisados com o auxílio do software Programa IBM-SPSS, versão 24.0 para Windows (SPSS, Inc., Chicago, IL, USA), atreladas as funcionalidades da ferramenta Excel®, versão 2016. Os quais foram submetidos a análise descritiva e inferencial. Foi aplicado o teste de normalidade Kolmogorov-Smirnov, e a Correlação de Spearman que objetivou a análise de predição linear entre as variáveis. Considerando-se nível de significância de $95 \%$ $(\mathrm{p}<0,05)$.

\section{Resultados}

A amostra desta pesquisa foi constituída na maioria por mulheres, idade entre 31 a 40 anos, tempo de profissão até 10 anos, tempo de experiência em Cuidados Paliativos (CP) até 1 ano e o hospital como local de trabalho. (Tabela 1).

Tabela 1. Caracterização dos profissionais da pesquisa. São José do Rio Preto, SP, Brasil. 2020.

\begin{tabular}{|c|c|c|c|c|c|c|}
\hline \multirow{2}{*}{ Informação } & \multicolumn{2}{|c|}{ Enfermeiros } & \multicolumn{2}{|c|}{$\begin{array}{l}\text { Auxiliares/técnicos } \\
\text { de enfermagem }\end{array}$} & \multicolumn{2}{|c|}{ TOTAL } \\
\hline & $\mathbf{N}$ & $\%$ & $\mathbf{N}$ & $\%$ & $\mathbf{N}$ & $\%$ \\
\hline \multicolumn{7}{|l|}{ Sexo } \\
\hline Feminino & 22 & 100,00 & 37 & 77,08 & 59 & 84,29 \\
\hline Masculino & 0 & 0,00 & 11 & 22,92 & 11 & 15,71 \\
\hline Total & 22 & 100,00 & 48 & 100,00 & 70 & 100,00 \\
\hline \multicolumn{7}{|l|}{ Faixa etária } \\
\hline Até 30 anos & 8 & 36,36 & 15 & 31,25 & 23 & 32,86 \\
\hline 31 a 40 anos & 8 & 36,36 & 19 & 39,58 & 27 & 38,57 \\
\hline 41 a 50 anos & 3 & 13,64 & 10 & 20,83 & 13 & 18,57 \\
\hline 51 a 60 anos & 3 & 13,64 & 2 & 4,17 & 5 & 7,14 \\
\hline$>60$ anos & 0 & 0,00 & 1 & 2,08 & 1 & 1,43 \\
\hline Missing & 0 & 0,00 & 1 & 2,08 & 1 & 1,43 \\
\hline Total & 22 & 100,00 & 48 & 100,00 & 70 & 100,00 \\
\hline \multicolumn{7}{|c|}{ Tempo de Profissão } \\
\hline Até 10 anos & 13 & 59,09 & 28 & 58,33 & 41 & 58,57 \\
\hline De 11 a 20 anos & 6 & 27,27 & 16 & 33,33 & 22 & 31,43 \\
\hline 21 a 30 anos & 2 & 9,09 & 4 & 8,33 & 6 & 8,57 \\
\hline Mais que 30 anos & 1 & 4,55 & 0 & 0,00 & 1 & 1,43 \\
\hline Total & 22 & 100,00 & 48 & 100,00 & 70 & 100,00 \\
\hline \multicolumn{7}{|c|}{ Tempo de Experiência em CP } \\
\hline Até 1 ano & 16 & 72,73 & 24 & 50,00 & 40 & 57,14 \\
\hline De 2 a 5 anos & 4 & 18,18 & 9 & 18,75 & 13 & 18,57 \\
\hline De 6 a 10 anos & 1 & 4,55 & 5 & 10,42 & 6 & 8,57 \\
\hline Mais que 10 anos & 1 & 4,55 & 2 & 4,17 & 3 & 4,29 \\
\hline Missing & 0 & 0,00 & 8 & 16,67 & 8 & 11,43 \\
\hline & & 4 & & & & \\
\hline
\end{tabular}




\begin{tabular}{lllllll} 
Total & $\mathbf{2 2}$ & $\mathbf{1 0 0 , 0 0}$ & $\mathbf{4 8}$ & $\mathbf{1 0 0 , 0 0}$ & 70 & $\mathbf{1 0 0 , 0 0}$ \\
Local de trabalho & & & & & & \\
UBSF & 12 & 54,55 & 5 & 10,42 & 17 & 24,29 \\
Hospital & 10 & 45,45 & 43 & 89,58 & 53 & 75,71 \\
Total & $\mathbf{2 2}$ & $\mathbf{1 0 0 , 0 0}$ & $\mathbf{4 8}$ & $\mathbf{1 0 0 , 0 0}$ & 70 & $\mathbf{1 0 0 , 0 0}$ \\
\hline
\end{tabular}

Fonte: Autores (2021).

A Tabela 2 demonstra que a maioria relatou experiência em processo de morrer, não possui curso específico em CP, é capaz de prestar $\mathrm{CP}$, relata que sabe a definição de $\mathrm{CP}$ e que sabe comunicar más notícias, mas também informa que não receberam informação suficiente sobre $\mathrm{CP}$.

Tabela 2. Autoavaliação sobre cuidados paliativos dos Enfermeiros e Auxiliares/Técnicos de enfermagem participantes da pesquisa. São José do Rio Preto, SP, Brasil. 2020.

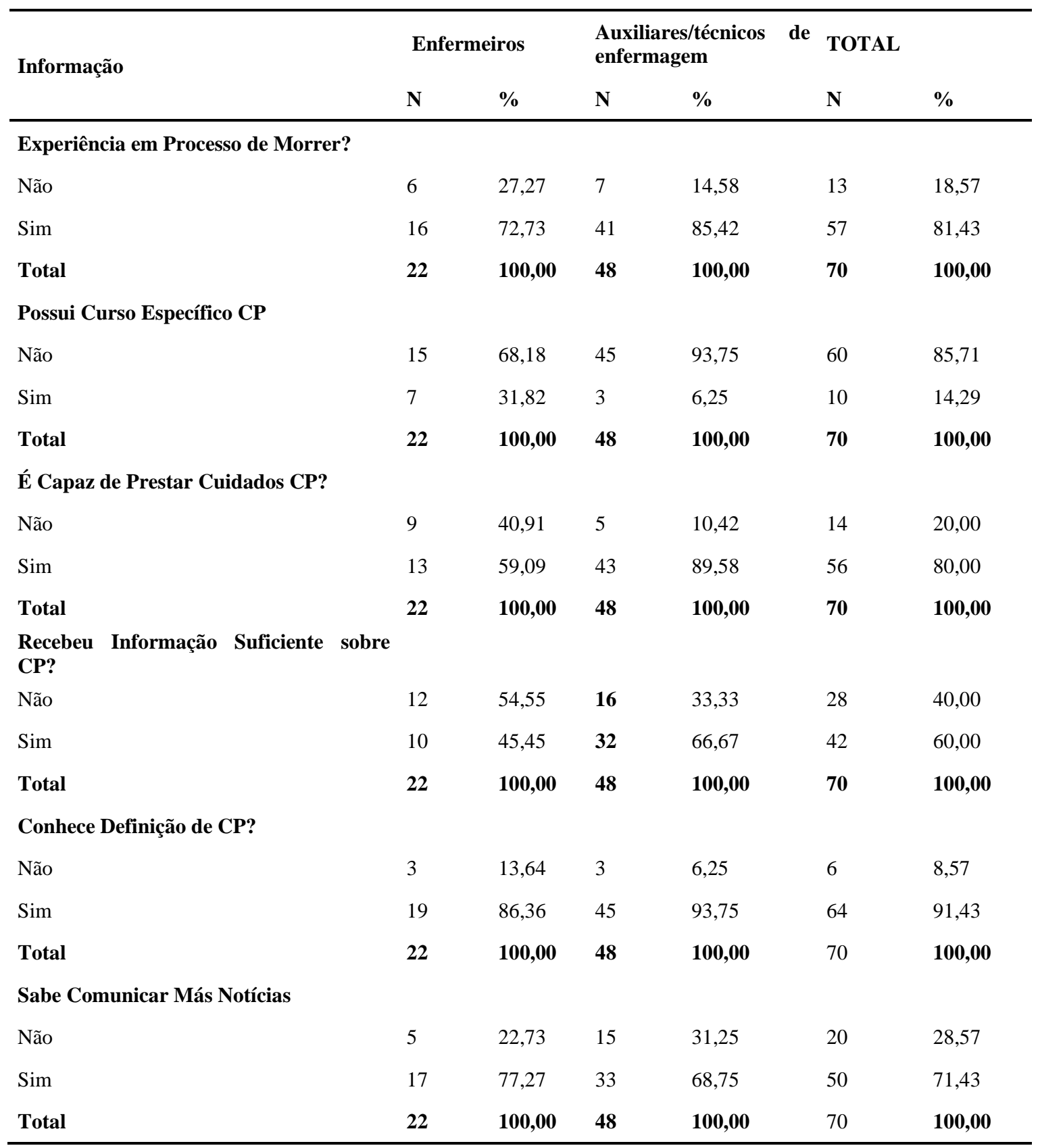


Fonte: Autores (2021).

Sobre as respostas relacionadas as definições de cuidados paliativos, eutanásia, distanásia e ortotanásia, a maior porcentagem de acertos foi na definição de cuidados paliativos e de erros foi na definição de distanásia (Tabela 3).

Ainda na Tabela 3 observa-se que mais da metade dos profissionais do hospital erraram a definição de distanásia. A resposta não sei para Distanásia e Ortotanásia foi maior entre os profissionais que trabalham na UBSF.

Tabela 3. Distribuição das respostas das definições de Cuidados paliativos, Eutanásia, Distanásia e Ortotanásia segundo as categorias dos profissionais da enfermagem, participantes do estudo e local de trabalho. São José do Rio Preto, SP, Brasil. 2020 .

\section{Enfermeiros}

Informação
$22(100 \%)$

\section{UBSF}

$\mathrm{n}=12(100 \%)$ $\mathrm{n}(\%)$

paliativos (Q1)

Acertos

Erros

Não Sei

Eutanásia (Q5)

Acertos

$10(83,33)$

Erros

Não Sei

Distanásia (Q6)

Acertos

Erros

Não Sei

Ortotanásia

(Q7)

\begin{tabular}{lccccc}
\hline $\begin{array}{l}\text { Cuidados } \\
\text { paliativos (Q1) }\end{array}$ & $\mathrm{n}(\%)$ & $\mathrm{n}(\%)$ & $\mathrm{n}(\%)$ & $\mathrm{n}(\%)$ & $\mathrm{n}(\%)$ \\
Acertos & $12(100,00)$ & 10 & $5(100,00)$ & $40(93,02)$ & $67(95,71)$ \\
Erros & $0(0,00)$ & $0(0,00)$ & $0(0,00)$ & $03(6,97)$ & $03(4,29)$ \\
Não Sei & $0(0,00)$ & $0(0,00)$ & $0(0,00)$ & $0(0,00)$ & $0(0,00)$ \\
Eutanásia (Q5) & & & & \\
Acertos & $10(83,33)$ & 10 & $04(80,00)$ & $37(86,04)$ & $61(87,14)$ \\
Erros & $0(0,00)$ & $0(0,00)$ & $0(0,00)$ & $02(4,65)$ & $02(2,86)$ \\
Não Sei & $02(16,66)$ & $0(0,00)$ & $01(20,00)$ & $04(9,30)$ & $07(10,00)$ \\
Distanásia (Q6) & & & & \\
Acertos & $04(33,33)$ & $04(40,00)$ & $0(00,00)$ & $09(20,93)$ & $17(24,29)$ \\
Erros & $02(16,66)$ & $05(50,00)$ & $0(0,00)$ & $22(51,16)$ & $29(41,43)$ \\
Não Sei & $06(50,00)$ & $01(10,00)$ & $05(100,00)$ & $12(27,90)$ & $24(34,29)$ \\
Ortotanásia & & & & \\
(Q7) & & & & & \\
Acertos & $05(41,66)$ & $07(70,00)$ & $03(60,00)$ & $31(72,09)$ & $46(65,71)$ \\
Erros & $01(8,33)$ & $01(10,00)$ & $0(0,00)$ & $01(2,32)$ & $03(4,29)$ \\
Não Sei & $06(50,00)$ & $02(20,00)$ & $02(40,00)$ & $11(25,58)$ & $21(30,00)$ \\
\hline
\end{tabular}

Fonte: Autores (2021).

\section{Auxiliares/ técnicos de}

enfermagem

$48(100 \%)$

\section{UBSF Hospitalar Total}

$\mathrm{n}=10 \quad \mathrm{n}=5(100 \%) \quad \mathrm{n}=43 \quad \mathrm{n}=70$

$(100 \%)$

$(100 \%) \quad(100 \%)$

Em geral a análise das respostas das questões sobre conhecimentos em cuidados paliativos revelou 1614 acertos (76,66\%), 240 erros (11,43\%) e 246 não souberam responder (11,71\%). 
Foi constatado que há tendência estatística entre o número de acertos com o tempo de experiência em CP ( $\mathrm{p}=0,005$ e corr $=0,333)$ e há tendência entre o número de acertos e aqueles que fizeram curso específico de $\mathrm{CP}(\mathrm{p}=0,020$ e corr $=0,277)$.

\section{Discussão}

O estudo foi realizado em um contexto de pandemia por Coronavírus -19 (COVID 19), período em que os profissionais da saúde estão com sobrecarga de trabalho, fadiga, ameaças a própria saúde, frustração por não conseguir salvar vidas e risco aumentado de ser infectado, adoecer e morrer (Schmidt et al., 2020). Isto pode ter colaborado para a não participação dos profissionais, percebida pelo não preenchimento de 48 questionários.

$\mathrm{O}$ fato de a maioria dos participantes ser do sexo feminino corrobora os dados de dois estudos, um realizado no centro-oeste paulista, em que os participantes do sexo feminino da equipe de enfermagem formavam 91,70\% do total (Costa \& Silva, 2021) e outro realizado no Rio de Janeiro, com a amostra de nove profissionais do sexo feminino de um total de 13 colaboradores (Morais et al., 2018). Isso enfatiza o contexto histórico e cultural que a profissão é inserida, uma vez que era caracterizada por representações femininas relacionadas a vocação do processo de cuidar, valorizada pelo modelo de Florence Nightingale, porém segregada com critérios de inclusão relacionada ao gênero, raça, necessidades de ter vocação para a arte de cuidar, ter formação educacional e religiosa, e de preferência cristã (Santos et al., 2020).

Verificou-se a predominância de profissionais com tempo de experiência até 1 ano, e o teste estatístico apontou que há tendência entre o número de respostas corretas com o tempo de experiência em CP. Esta relação é ressaltada por outro estudo realizado em seis países europeus, em que o melhor nível de conhecimento sobre cuidados paliativos foi influenciado pelo número de anos de experiência, uma vez que profissionais com mais de 10 anos de experiência tinham mais conhecimentos do que aqueles com menos anos (Smets et al., 2018).

Constatou-se que 76,66\% dos profissionais das Unidades Hospitalares e UBSF acertaram as questões de conhecimentos gerais sobre cuidados paliativos, o que pressupõe que há um domínio sobre o tema de cuidados paliativos, mas não completo, assim como demais estudos realizados com profissionais de enfermagem em unidades de internação, urgências e unidades de pacientes críticos que mostraram conhecimentos suficientes sobre cuidados paliativos, com uma porcentagem de acertos de 54,00\% (Chover-Sierra, Martínez-Sabater, \& Lapeña-Moñux, 2017), e em enfermarias de oncologia, terapia intensiva e cardiologia com uma porcentagem média de respostas corretas de 67,60\% (Autor, Storey, \& Ziemba-Davis., 2013).

Por outro lado, um estudo realizado com estudantes de enfermagem na China, no ano de 2020 demonstrou que os acadêmicos apresentavam baixo nível de conhecimento em CP (Zhou, \& Zhang, 2021). Com isso, pressupõe que adequações da educação técnica e superior sobre o tema são necessárias, o que é ressaltado em outro estudo realizado no Brasil com acadêmicos de enfermagem, onde a maioria dos estudantes afirmou que não teve contato com nenhum componente curricular que abordasse diretamente o tema. Além disso, o estudo também destaca a necessidade de aprofundar os conhecimentos acerca da temática, uma vez que há limitações sobre as experiências vivenciadas e poucas oportunidades de se estabelecer discussões durante a graduação, resultando em um despreparo na assistência aos pacientes em CP (Guimarães et al, 2020).

Observou-se que o maior número de respostas erradas ou que não souberam responder corresponde aos conceitos de distanásia, seguido de ortotanásia. Esta lacuna é percebida em uma porcentagem maior entre os técnicos e auxiliares de enfermagem. Estes resultados sugerem um déficit destes conhecimentos básicos, que podem ser abordados desde a graduação e/ou curso técnico. Neste âmbito entra em discussão que a falta de conhecimento sobre definições que englobam o tema é percebida não apenas na equipe de enfermagem, a qual presta os cuidados diretos ao paciente, mas também na equipe multidisciplinar necessária para o CP. Estudo desenvolvido com enfermeiros, médicos e fisioterapeutas mostrou que pelo menos um representante de cada categoria profissional não soube responder o conceito de eutanásia, ortotanásia e distanásia (Nascimento et al., 2020). Nesse contexto torna-se necessário refletir que a dificuldade encontrada em diferenciar estes 
conceitos pode implicar na qualidade da assistência prestada, uma vez que o conhecimento direciona as decisões a serem tomadas, que são princípios éticos fundamentais de responsabilidade de todos os profissionais de saúde que visam a qualidade de vida no CP.

Com referência a afirmação sobre informação suficiente sobre Cuidados Paliativos, 40,00\% dos participantes responderam que não receberam informações suficientes. Este resultado indica que mesmo o estudo demonstrando que há uma porcentagem satisfatória de conhecimentos gerais de toda a equipe de enfermagem sobre cuidados paliativos, há uma porcentagem significativa de informações insuficientes, o que pode contestar os conhecimentos se analisado de forma integral. Pressupõe que isso se deve ao contato vivenciado na prática da assistência, mas ainda há uma deficiência na fundamentação do conhecimento, o que pode implicar em uma assistência muitas vezes ineficaz para cuidados paliativos.

A partir dos resultados apresentados anteriormente, é fundamental que ocorra o fortalecimento do acesso ao ensino sobre CP, tanto na graduação, como em capacitações realizadas com os profissionais da equipe de CP dentro da instituição. Destaca-se que é importante realizar educação em saúde continuada, em diferentes níveis de complexidade, uma vez que a assistência em CP deve partir de todos os níveis de atenção, visto que o CP pode estar presente em todos os ciclos da vida.

Salienta-se que $85,71 \%$ dos profissionais afirmaram não possuir cursos específicos em cuidados paliativos e $68,18 \%$ dos enfermeiros não possuem. Isto implica diretamente na equipe de enfermagem, uma vez que o enfermeiro deve desenvolver competências que incluem a coordenação da equipe, liderança, capacitação e desenvolvimento profissional. Um estudo realizado na Espanha, com 159 profissionais de enfermagem apresentou resultados divergentes da atual pesquisa, visto que uma porcentagem importante de $64,20 \%$ indicou ter recebido formação em cuidados paliativos durante a graduação e apenas 24,00\% indicou não possuir formação ou experiência na área (Chover-Sierra et al., 2017). Essa discordância pode ser decorrente de populações diferentes, uma vez que o atual estudo foi realizado em um Hospital no interior de São Paulo, no Brasil e o outro estudo em Hospital Geral Universitário de Valência na Espanha. Dessa forma, compreende-se que a oferta de condições apropriadas para a atuação da equipe de enfermagem se faz necessária por meio de diversos recursos, incluindo treinamento ou capacitações específicas para um conhecimento ampliado sobre a especialidade.

Em concordância, nota-se que há um prejuízo na qualidade de vida dos pacientes, em que se percebe a falta de um olhar mais humanizado voltado ao ser biopsicossocial e suas necessidades espirituais (Albuquerque et al., 2018). Diante disto, fica evidente a relevância de criação de vínculo entre profissional, usuário e família, a fim de não potencializar os aspectos relacionados à doença, mas sim a qualidade na finitude da vida que é um dos princípios dos CP. Ressalta-se que uma abordagem adequada pode ter como facilitador o desenvolvimento contínuo da competência de comunicação pelo profissional, sendo que não é esperado o alcance do conhecimento completo, mas sim um aperfeiçoamento das habilidades nos processos de cuidar e relações (Dalcól, et al., 2018).

Esta habilidade está relacionada a assertividade na comunicação de más notícias, que culturalmente, é uma atribuição destinada ao profissional médico, porém, o enfermeiro por meio de sua autonomia pode exercer essa função. No atual estudo $71,43 \%$ dos profissionais afirmaram que sabem comunicar más notícias, entretanto um estudo realizado com 21 enfermeiros que atuavam no pronto atendimento de uma cidade do Centro-Oeste de Minas Gerais contradiz este resultado, pois apontam um despreparo e desconhecimento desses profissionais relacionado a vivência ao comunicar más notícias, além de atribuir a função ao outro profissional (Silva et al, 2020). Isto pode ser explicado devido às diferenças na abordagem metodológica, uma vez que o atual estudo foi de natureza quantitativa e o outro qualitativo de caráter descritivo.

Acerca das competências e técnicas listadas, destaca-se que a equipe de enfermagem tem papel fundamental ao viabilizar o cuidado, o processo de saúde e doença e as atitudes para lidar com a terminalidade da vida. Entretanto, para que ocorra o fortalecimento destas ações, é importante uma coordenação em âmbito interdisciplinar, priorizando as necessidades biopsicossociais e espirituais individuais do paciente e sua família em CP. 
Outro estudo, realizado na China, em 2016 concluiu que o conhecimento dos estudantes chineses de graduação em enfermagem sobre CP é mínimo. Situação que sugere o desenvolvimento de um programa eficaz de cuidados no final da vida para estudantes de enfermagem, pois com o envelhecimento da população e o aumento do número de sobreviventes das doenças crônicas como o câncer, os cuidados paliativos será futuramente um problema de saúde pública global. Os autores ainda acrescentam que como uma força central para o desenvolvimento sustentável do campo da enfermagem, o conhecimento e as atitudes dos alunos de graduação em enfermagem sobre os cuidados paliativos afetarão diretamente a qualidade do atendimento aos pacientes terminais no futuro (Jiang et al, 2019).

Este estudo teve como limitações o período pandêmico por COVID-19 e a dificuldade da adesão dos enfermeiros e técnicos/auxiliares na participação e no preenchimento dos questionários, mas compreende-se que é um ponto de partida para outros estudos de natureza qualitativa, assim como a possibilidade de repensar a introdução do conteúdo de CP na grade curricular dos cursos de formação.

\section{Conclusão}

Com este estudo foi possível observar que aspectos básicos do cuidado paliativo devem fazer parte da educação em serviço, ou seja, é importante que os profissionais entendam que ações devem ser implementadas ao paciente em CP e que o plano de cuidados não tem como objetivo abreviar a vida ou causar sofrimento, mas oferecer qualidade de vida até o momento da morte. Acredita-se que esta pesquisa pode contribuir para uma reflexão sobre o ensino-aprendizagem e a formação do profissional da enfermagem com a finalidade de oferecer um cuidado ideal ao paciente com uma doença sem possibilidade de cura.

\section{Referências}

Albuquerque, M. B., França, M. S., Fonseca, A. C. T., Azeredo, M. G., Oliveira, R. K., Silva, B. O., \& Pinheiro, I. F. (2018). Qualidade na assistência de cuidados paliativos em idosos. CIPEEX. 2, 1812-1815. http://anais.unievangelica.edu.br/index.php/CIPEEX/article/view/2851/1586

Autor, S. H., Storey, S. L., \& Ziemba-Davis, M. (2013). Knowledge of Palliative Care: An Evaluation of Oncology, Intensive Care, and Heart Failure Nurses. Journal of Hospice \& Palliative Nursing, 15(5), 307-315. https://doi.org/10.1097/njh.0b013e3182930800

Resolução $n^{\circ} 41$, de 31 outubro de 2018. Dispõe sobre as diretrizes para a organização dos cuidados paliativos, à luz dos cuidados continuados integrados, no âmbito do Sistema Único de Saúde. https://www.in.gov.br/materia/-/asset_publisher/Kujrw0TZC2Mb/content/id/51520746/do1-2018-11-23-resolucao-n-41de-31-de-outubro-de-2018-51520710

Cano, C. W. A., Silva, A. L. C., Barboza, A. F., Bazzo, B. F., Martins, C. P., Iandoli Júnior, D., Benites, L. S. B., Terceros, L. B., \& Nantes, R. S. G.(2020). Finitude da vida: compreensão conceitual da eutanásia, distanásia e ortotanásia. Rev. Bioét. 28 (2), 376-8. http://dx.doi.org/10.1590/1983-80422020282399

Chover-Sierra, E., Martínez-Sabater, A., \& Lapeña-Moñux, Y. (2017). Conhecimentos em cuidados paliativos dos profissionais de enfermagem de um hospital espanhol. Rev. Latino-Am. Enfermagem. 25, e2847. https://doi.org/10.1590/1518-8345.1610.2847.

Côbo, V. A., Dal Fabbro, A. L., Parreira, A. C. S. P., \& Pardi, F. (2019). Cuidados Paliativos na Atenção Primária à Saúde: perspectiva dos profissionais de saúde. Boletim - Academia Paulista de Psicologia, 39(97), 225-235. http://pepsic.bvsalud.org/scielo.php?script=sci_arttext\&pid=S1415711 X2019000200008\&lng=pt\&tlng=pt.

Costa, B. M., \& Silva, D. A. (2021). Atuação da equipe de enfermagem em cuidados paliativos. Research, Society and Development, $10(2)$, e28010212553. http://dx.doi.org/10.33448/rsd-v10i2.12553

D’Alessandro, M. P. S., Pires, C. T., Forte, D. N., Messias, A. A., Maiello, A. P. M. V., Coelho, F. P., Schuls, V. (Coord). (2020). Manual de Cuidados Paliativos/São Paulo: Hospital Sírio Libanês; Ministério da Saúde, 13-22.

Dalcól, C., Garanhani, M. L., Fonseca, L. F., \& Carvalho, B. G. (2018) Competência em comunicação e estratégias de ensino-aprendizagem: percepção dos estudantes de enfermagem. Cogitare Enferm, 23(3), e53743. https://dx.doi.org/10.5380/ce.v23i3.53743

Guimarães, J. A. M., Dantas, R. R, Bezerra, T. A., Medeiros, A. C. T., \& Medeiros, F. A. L (2020). Nursing students' perceptions of palliative care. Rev Rene, 21, e44033. https://doi.org/ 10.15253/2175-6783.20202144033

Jiang, Q., Lu, Y., Ying, Y., \& Zhao H. (2019) Attitudes and knowledge of undergraduate nursing students about palliative care: An analysis of influencing factors. Nurse Educ Today, Sep, 80, 15-21. https://doi.org/10.1016/j.nedt.2019.05.040. 
Matsumoto, D. Y. (2009). Cuidados Paliativos: conceito, fundamentos e princípios. In: Academia Nacional de Cuidados Paliativos (org). Manual de cuidados paliativos. Diagraphic, 14-19.

Morais, E. N., Conrad, D., Mattos, E. M., Cruz, S. A. C, Machado, G. C, \& Abreu, M. O. (2018). Cuidados paliativos: enfrentamento dos enfermeiros de um hospital privado na cidade do Rio de Janeiro - RJ. Rev Fund Care Online, 10(2), 318-325. http://dx.doi.org/10.9789/2175-5361.2018.v10i2.318-325

Mota, A. E. Z., Santos, D. P. M., Pertile, A. C., Nodari, P. R. G., Santos, T. M., \& Miranda, E. B. (2021). A percepção dos profissionais de saúde sobre os aspectos jurídicos e conceituais da eutanásia. Brazilian Journal of Development, 7(2),17773-17788. http://doi.org/10.34117/bjdv7n2-427

Nascimento, L. A., Sousa, N. M., Santos, A. C. B. C., Sousa, M. N., Assis, E. V., \& Pascoal, K. P. M. F. (2020). Avaliação do conhecimento dos profissionais de saúde sobre tratamentos paliativos em unidade de terapia intensiva. Revista facisa on-line. 9(2), 94-105. http://webcache.googleusercontent.com/search?q=cache:wDlf0QvFh5wJ:periodicos.unicathedral.edu.br/revistafacisa/article/download/572/432+\&cd=2\&hl=pt $-\mathrm{BR} \& \mathrm{ct}=\mathrm{clnk} \& \mathrm{gl}=\mathrm{br}$

Santos, F. B. O., Rabelo, A. R. M., França, B. D, Carregal, F. A. S, Marques, R. C., \& Silva, K. L. (2020). Black women in nursing history: the cultural competence in Maria Barbosa Fernandes' trajectory. Rev. Bras. Enferm, 73(4), e20190221. https://doi.org/10.1590/0034-7167-2019-0221

Schmidt, B., Crepaldi, M. A., Bolze, S. D. A., Neiva-Silva, L., \& Demenech, L. M. (2020). Saúde mental e intervenções psicológicas diante da pandemia do novo coronavírus (COVID-19). Estudos de Psicologia (Campinas), 37, e200063. http://doi.org/10.1590/1982-0275202037e200063.

Silva, A. E., Ribeiro, A. S., Carvalho, T. V., Laia, D. H. S., Ferreira, G. J., \& Oliveira L. A. (2020). A percepção do profissional enfermeiro frente à comunicação de notícias difíceis. Pesquisa, Sociedade e Desenvolvimento 9(12), e36991211014. ttps://doi.org/10.33448 / rsd-v9i12.11014.

Silva, C. P., Santos, A. T. C., Silva, R. P., Andrade, J. D., \& Almeida, L. M. (2016). Significado dos Cuidados Paliativos para a Qualidade da Sobrevivência do Paciente Oncológico. Revista Brasileira de Cancerologia, 62(3), 225-235. https://rbc.inca.gov.br/site/arquivos/n_62/v03/pdf/05-artigo-significado-doscuidados-paliativos-para-a-qualidade-da-sobrevivencia-do-paciente-oncologico.pdf

Silva, E. M. (2018). A Ortotanásia e o direito de morrer a luz do princípio da dignidade da pessoa humana.Jus.com.br. https://jus.com.br/artigos/69887/aortotanasia-e-o-direito-de-morrer-a-luz-do-principio-da-dignidade-da-pessoa-humana

Silva, R. S. (2021). A enfermagem em cuidados paliativos. Academia nacional de cuidados paliativos (ANCP). https://paliativo.org.br/a-enfermagem-emcuidados-paliativos/

Smets, T., Pivodic, L., Piers, R. W., Pasman, H. R, Engels, Y., Szczerbińska K., Kylänen, M., Gambassi, G., Payne, S., Deliens, L., \& Block L. V. (2018) The palliative care knowledge of nursing home staff: The EU FP7 PACE cross-sectional survey in 322 nursing homes in six European countries. Palliat Med, 32(9), 1487-1497. https://doi.org/10.1177/0269216318785295

Steel, J. L., Geller, D.A., Kim, K. H., Butterfield, L.H., Spring, M., Grady, J., Sun, W*, Marsh, w., Antoni, M., Dew. M. A., Helgeson, V., Schulz, R., \& Tsung, A. (2016). Web-based collaborative care intervention to manage cancer-related symptoms in the palliative care setting. Cancer, $122(8)$, $1270-1282$. http://doi.org/10.1002/cncr.29906. Epub 2016 Mar 11.

World Health Organization. (2014). Worldwide Palliative Care Aliance. Global Atlas of Palliative Care at the End of Life, 2014. https://www.who.int/nmh/Global_Atlas_of_Palliative_Care.pdf.

Zhou, Y., Li, Q., \& Zhang, W. (2021). Undergraduate nursing students' knowledge, attitudes and self-efficacy regarding palliative care in China: A descriptive correlational study, 8(1), 343-353. https://doi.org/10.1002/nop2.635 